Assumptions 1, 2 are relaxed. Thanks to their constructive nature, the conditions obtained can be easily verified. Since the Assumptions assumed in this paper are weaker than the known from literature constraint qualifications for SIP problems, the new optimality conditions can be applied for a more general case of SIP problems.

\title{
References
}

1. Goberna M.A., Lópes M.A. Semi-Infinite Programming: recent advances. Kluwer, Dordrecht. 2001.

2. Ruckmann J.-J., Shapiro A. Second-order optimality conditions in Generalized Semi-Infinite Programming// Set-Valued Analysis. 2001. No. 9. P. 169-186.

3. Kostyukova O.I., Tchemisova T.V. Implicit Optimality Criterion for convex SIP problem with box constrained index set// TOP. 2012. V. 20, No. 2, P. 475-502.

4. Mangasarian O.L., Formovitz S. The Fritz-John necessary optimality conditions in presence of equality and inequality constraints// J. Math. Anal. Appl. 1967. V. 17. P. $37-47$.

\section{NONLINEAR POSITIONAL DIFFERENTIAL GAME IN THE CLASS OF MIXED STRATEGIES}

\author{
A.N. Krasovskii \\ Ural State Agricultural University \\ Karl Liebknecht Str. 42, Ekaterinburg, 620075, Russia \\ ankrasovskii@gmail.com
}

The feedback control problem for a nonlinear dynamic system under lack of information on disturbances is considered. The problem on minmaxmaxmin of ensured result for a given positional quality index is formalized into an antagonistic two-player differential game in the framework of the concept of the Sverdlovsk (Ekaterinburg) school on the theory of control and differential games. The problem is solved in the class of mixed positional strategies. The existence of a solution for considered differential game - of the value of the game and the saddle point - is determined. The solution of a problem is based on application of the appropriate modelsleaders, the so-called methods of minimax and maximin extremal shift [2] and the method of upped convex hulls [1]. Although we use probabilistic mechanisms in formation of control, the final result is guaranteed with probability arbitrary close to one. Results of the study are applied to the control model [3] of a mechanical device. It simulates a controller in the space equipment used for docking and landing of modules. Simulation outputs are presented. 


\section{References}

1. Krasovskii A.N., Krasovskii N.N. Control under Lack of Information. Boston, Birkhauser, 1994.

2. Krasovskii A.N., Choi Y.S. Stochastic Control with the Leaders-Stabilizers. Ekaterinburg, Institute of Mathematics and Mechanics UrB RAS, 2001.

3. Krasovskii A.A., Krasovskii A.N. Nonlinear positional differential game in the class of mixed strategies // Proceedings of the Steklov Institute of Mathematics, Vol. 277, 2012. P. 137-143.

\section{SUBDIFFERENTIAL SLOPES AND STABILITY OF ERROR BOUNDS FOR CONVEX CONSTRAINT SYSTEMS}

\section{A.Y. Kruger ${ }^{1}$, Huynh Van Ngai ${ }^{2}$, M. Théra ${ }^{3}$}

${ }^{1}$ Centre for Informatics and Applied Optimisation

School of Science, Information Technology and Engineering, University of Ballarat

POB 663, Ballarat, Vic, 3350, Australia

a.kruger@ballarat.edu.au

2 Department of Mathematics, University of Quynhon,

170 An Duong Vuong, Qui Nhon, Vietnam

nghiakhiem@yahoo.com

${ }^{3}$ Laboratoire XLIM, UMR-CNRS 6172, Université de Limoges, France michel.thera@unilim.fr

1. Error Bounds. Given a function $f: X \rightarrow \mathbb{R}_{\infty}:=\mathbb{R} \cup\{+\infty\}$ on a Banach space $X$ and a point $\bar{x} \in X$ with $f(\bar{x})=0$, we say that $f$ admits a (local) error bound at $\bar{x}$ if there exist reals $c>0$ and $\delta>0$ such that

$$
c d\left(x, S_{f}\right) \leq[f(x)]_{+} \text {for all } x \in B_{\delta}(\bar{x}),
$$

where $S_{f}:=\{x \in X: f(x) \leq 0\}$ and the notation $\alpha_{+}:=\max (\alpha, 0)$ is used, or equivalently

$$
\operatorname{Er} f(\bar{x}):=\liminf _{x \rightarrow \bar{x}, f(x)>0} \frac{f(x)}{d(x, S(f))}>0 .
$$

2. Subdifferential Slopes. From now on, $f: X \rightarrow \mathbb{R}_{\infty}$ is a proper lower semicontinuous convex function on a Banach space $X$ and $f(\bar{x})<\infty$. Recall the definition of the subdifferential of $f$ at $\bar{x}$ :

$$
\partial f(\bar{x})=\left\{x^{*} \in X^{*} \mid f(x)-f(\bar{x}) \geq\left\langle x^{*}, x-\bar{x}\right\rangle, \forall x \in X\right\} .
$$

The subdifferential slope, boundary subdifferential slope, and strict outer subdifferential slope of $f$ at $\bar{x}$ are defined as follows:

$$
\begin{aligned}
|\partial f|(\bar{x}) & =\inf \left\{\left\|x^{*}\right\| \mid x^{*} \in \partial f(\bar{x})\right\}, \\
|\partial f|_{b d}(\bar{x}) & =\inf \left\{\left\|x^{*}\right\| \mid x^{*} \in \operatorname{bd} \partial f(\bar{x})\right\}, \\
|\partial f|^{>}(\bar{x}) & =\liminf _{x \rightarrow \bar{x}, f(x) \downarrow f(\bar{x})}|\partial f|(x) .
\end{aligned}
$$

